\title{
Nanofiltration performance of conical and hourglass nanopores
}

\author{
Béatrice Balannec $^{1}$, Aziz Ghoufi ${ }^{2}$, Anthony Szymczyk $^{1 *}$ \\ ${ }^{1}$ Univ Rennes, Ecole Nationale Supérieure de Chimie de Rennes, CNRS, ISCR (Institut des \\ Sciences Chimiques de Rennes) - UMR 6226, F-35000 Rennes, France \\ ${ }^{2}$ Univ Rennes, CNRS, IPR (Institut de Physique de Rennes) - UMR 6251, F-35000 Rennes,
} France

*E-mail: anthony.szymczyk@univ-rennes1.fr

\begin{abstract}
Pressure-driven ion transport through conical and hourglass-shape (double conical) nanopores is investigated theoretically by solving the Poisson, Nernst-Planck and Navier-Stokes equations numerically. Due to the electrostatic asymmetry in conical nanopores, these latter exhibit nanofiltration rectification properties, i.e. they reject salts differently depending if the solution enters the nanopore from its base or its tip. The filtration rectification properties of conical nanopores result from two different phenomena, (i) the co-ion exclusion at the pore mouth and (ii) the sign and the magnitude of the (pressure-induced) electric field arising through the nanopores. Hourglass-shape nanopores exhibit improved separation performance compared with cylindrical and conical nanopores with identical average diameter, length and surface charge density. Notably, they allow target salt rejections to be reached with a smaller driving force than the other pore geometries, which makes them attractive candidates for the design of advanced nanofiltration membranes allowing less energy intensive separations.
\end{abstract}




\section{Graphical abstract:}

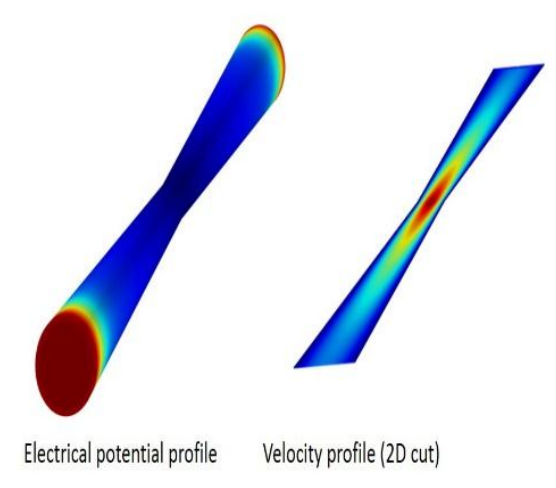

\section{Introduction}

Ion transport in synthetic nanochannels has become a field of intensive research thanks to the remarkable advances in fabrication technology [1-8]. Nanochannels are characterized by a high surface-to-volume ratio and dimensions comparable with the Debye length, thus leading to surface-charge-governed ion transport with direct implications on e.g. their conductance, selectivity or efficiency in electrokinetic energy conversion [9-11].

Interestingly, nanopores exhibiting an electrostatic asymmetry possess rectification properties, i.e. the transport rate of ions depends on the direction of the driving-force applied through the nanopores [12-20]. For instance, Karnik et al. built an ionic diode, rectifying ion current in a similar way as a semiconductor diode rectifies electron current, by introducing a surface charge discontinuity in a nanofluidic channel [13]. Cheng and Guo reported rectified ion transport properties of silica nanochannels induced by concentration gradients [15] while Szymczyk et al. investigated pressure-induced salt flux rectifications by nanopores exhibiting 


\section{ACCEPTED MANUSCRIPT}

a symmetry breaking in the surface charge distribution with potential applications in water desalination $[17,18]$.

Conical nanopores, which can be obtained by track-etching techniques [21], are particularly attractive since their asymmetry in shape can lead to high current-voltage $(I-V)$ rectification degrees even in the case of homogeneously charged pores [22, 23]. The reason is that an heterogeneity in the pore shape leads to an heterogeneous distribution of the local volume charge density even if the surface charge is smeared homogenously over the pore walls. It was shown that the $I-V$ rectification properties of conical nanopores could be even improved by an appropriate surface functionalization, notably by creating bipolar pores with separate positively and negatively charged regions [24].

Thanks to their asymmetric transport properties conical nanopores are now considered as promising candidates in applications like nanofluidic diodes, ion pumps or sensors for chemical analysis and particle size detection [13, 24-30].

However, most reported works about rectification properties of conical nanopores have focused on electrically-driven ion transport while pressure-driven transport has not yet received attention although pressure-driven techniques like nanofiltration have gained increasing attention in recent years as environmentally friendly and energy efficient separation processes [19]. The aim of this work is then to investigate theoretically the nanofiltration performance of conical nanopores, including double conical pores with an hourglass-shape. Flux and rejections of various electrolytes (symmetric and asymmetric) are computed by means of the Poisson-Nernst-Planck theory combined with the Navier-Stokes equation and are compared with those obtained with a cylindrical benchmark nanopore.

\section{Theoretical model}

Four different pore shapes were considered in this work (see Figure 1): cylindrical, conicalconvergent (i.e. with the pore base facing the high pressure compartment), conical-divergent 


\section{ACCEPTED MANUSCRIPT}

(i.e. with the pore tip facing the high pressure compartment), and hourglass-shaped. The geometrical features of the different pores are collected in Table 1. The structural features of the different pores were chosen to be relevant with those of actual nanofiltration membranes [19]. Indeed, Bowen and Mohammad reported the mean pore diameter of twenty-nine commercial nanofiltration membranes in the range $0.8-3.2 \mathrm{~nm}$ [31]. We considered isothermal systems (with temperature $T$ set to $298 \mathrm{~K}$ ) and the external solutions in both high pressure and low pressure compartments were assumed to be ideal and perfectly stirred.

In this work a continuum-based approach was used to solve numerically the problem of pressure-driven transport of electrolytes through the various nanopores described in Table 1. This approach relies on the Poisson and Nernst-Planck equations to determine the electrical potential and the ion concentrations inside the nanopore, respectively:

$$
\begin{aligned}
& \nabla^{2} \psi=-\frac{\rho_{e}}{\varepsilon_{0} \varepsilon_{r}}=-\frac{F}{\varepsilon_{0} \varepsilon_{r}} \sum_{i} c_{i} z_{i} \\
& \mathbf{j}_{i}=-D_{i} \nabla c_{i}-\frac{F z_{i} D_{i} c_{i}}{R T} \nabla \psi+c_{i} \mathbf{u}
\end{aligned}
$$

where $\psi$ is the electrical potential, $\rho_{e}$ the space charge density, $F$ the Faraday constant, $\varepsilon_{0}$ the vacuum permittivity, $\varepsilon_{\mathrm{r}}$ the solution dielectric constant, $c_{i}, z_{i}, \mathbf{j}_{i}$ and $D_{i}$ are the concentration inside the nanopore, the charge number, the molar flux and the diffusion coefficient of the ion $i$, respectively, $R$ is the ideal gas constant, $T$ the temperature, and $\mathbf{u}$ is the fluid velocity resulting from the application of an external pressure gradient (the third term in Equation 2 represents the contribution of convection (or advection) to the ionic flux).

At steady state the ionic fluxes satisfy the continuity equation:

$$
\nabla \mathbf{j}_{i}=0
$$

The fluid velocity (u) in Equation 2 obeys the Navier-Stokes equation: 
$-\nabla P+\eta \nabla^{2} \mathbf{u}-F \sum_{i} c_{i} z_{i} \nabla \psi=\rho(\mathbf{u} \cdot \nabla) \mathbf{u}$

where $P$ is the hydrostatic pressure and $\eta$ and $\rho$ are the fluid viscosity and density, respectively.

The right-hand side of Equation 4 represents the inertia term which was found to be negligible in all simulations, as expected because of the low Reynold numbers under consideration.

For an incompressible fluid the continuity equation reads as follows:

$\nabla \mathbf{u}=0$

Due to axial symmetry the above system of three-dimensional equations is reduced into a two-dimensional problem. The following boundary conditions were specified in order to solve Equations 1-5:

-at the pore inlet $(z=0 \mathrm{~nm})$ : laminar parabolic inflow with average velocity $\mathbf{u}_{\text {inlet }}$ (input parameter), $\psi=0 \mathrm{mV}$ (by convention) and $c_{i}=c_{i, \text { inlet }}$ (input parameter) where $c_{i, \text { inlet }}$ is the concentration of the ion $i$ at the position $z=0$ (see Figure 1).

-at the pore axis: axial symmetry

-at the pore wall: the pore wall was considered to be ion-impenetrable $\left(\mathbf{n} \cdot \mathbf{j}_{i}=0\right.$, where $\mathbf{n}$ is the unit outer normal vector of the pore wall) and to bear a constant surface charge density $\sigma$ $\left(\sigma=-\varepsilon_{0} \varepsilon_{r} \mathbf{n} \cdot \nabla \psi\right)$. The no-slip condition was applied at the pore wall $(\mathbf{u}=0)$.

-at the pore outlet $(z=100 \mathrm{~nm}): P=0$ bar (by convention), outflow condition $\left(-\mathbf{n} \cdot D_{i} \nabla c_{i}=0\right)$ and $\psi=\psi_{\text {outlet }}$ (initially unknown; $\psi_{\text {outlet }}$ is estimated through an iterative process by taking into account the electroneutrality condition, i.e. $\sum_{i} c_{i, \text { outlet }} z_{i}=0$ where the $c_{i, \text { outlet }}$ values are initially unknown). 


\section{ACCEPTED MANUSCRIPT}

Due to their finite size, ions cannot come closer to the pore wall than to an effective distance of minimum approach, which was considered here as equal to their Stokes radius.

Equations 1-5 were solved simultaneously by a finite element method with the COMSOL Multiphysics 4.1 Software using the fully coupled direct solver MUMPS. The twodimensional, axisymmetric pores were meshed with very fine elements, especially in the inlet and outlet regions where strong concentration and electric potential gradients occurred. The mesh independence of the numerical solution was carefully checked. Depending on the pore shape, 20000 to 100000 elements were used for meshing (mostly triangular elements and quadrilateral elements for the boundary layers). The mesh statistics showed that the minimum element quality was around 0.3 and the average element quality higher than 0.9 (for highly anisotropic systems like the nanopores under consideration in the present work, a mesh quality above 0.1 is advisable).

The use of continuum-based theories to describe ion transport through nanopores has been a matter of controversy, notably because they cannot describe confinement-induced phenomena like e.g. the layering of solvent molecules confined in a nanochannel $[32,33]$ or the single-file arrangement of water molecules through subnanometer carbon nanotubes [34]. Nevertheless, approximate models based on continuum approaches have been found to account fairly well for experimental results obtained with pores of nanometric dimensions [22, 35, 36]. Regarding continuum hydrodynamics, the Navier-Stokes equation has been found to be remarkably robust, remaining valid down to extremely small length scales (typically $1 \mathrm{~nm}$ for water) $[37,38]$. Ható et al. showed that approximate models based on continuum theories can capture the overall physics of nanopores-based devices correctly (despite the incomplete description of molecular level phenomena) because they include the physics that is necessary from the point of view of the system transfer function, i.e. the relation between the input signal and the system response [20]. Therefore, continuum-based models giving at least a semi-quantitative description of ion transport can help to optimize the construction of 


\section{ACCEPTED MANUSCRIPT}

nanopores-based devices with desired transport characteristics [12]. It is also worth stressing that, in many cases, continuum-based models are the only way to compare with experiments because fully atomistic simulations remain too computationally expensive. For instance, the typical transmembrane pressure difference applied through nanofiltration membranes lies in the range 10-50 bar, which is far too small to get any statistically meaningful results from molecular simulations. Moreover, previous works suggested that the salt flux (and so the salt rejection) could vary monotonously or non-monotonously with the applied pressure difference depending on the surface charge distribution $[17,18]$. Since the aim of the present work was to investigate the nanofiltration performance of conical nanopores it was therefore essential to work with tools allowing actual nanofiltration operating conditions to be reproduced, i.e. $\Delta P \leq$ 50 bar. It is worth mentioning that the Poisson-Nernst-Planck theory has been found to fairly well describe experimental data for electrically-driven transport $[1,14,21,22,39]$ and concentration-gradient-driven transport [25, 40, 41] through conical nanopores. As stated above pressure-driven ion transport through conical nanopores has not yet received attention and so no direct comparison with experiment is possible at this time. However, models based on the Poisson, Nernst-Planck and Navier-Stokes equations (and considering cylindrical pores) has already been successfully confronted with transport experiments [13, 35, 42-44] and it seems reasonable to assume that the performance of this theoretical framework for describing electrically-driven transport and pressure-driven transport through conical pores should be similar.

\section{Results and discussion}

Figure 2 shows the variation of the salt rejection rate, defined as $100\left(1-c_{i, \text { outlet }} / c_{i, \text { inlet }}\right)$, as a function of the driving force $(\Delta P)$ applied through four different nanopores (pores A-D; see Figure 1 and Table 1 for pore shape and structural features, respectively). The surface charge 


\section{ACCEPTED MANUSCRIPT}

density $(\sigma)$ was set to $-1 \mathrm{mC} \mathrm{m}^{-2}$ and a $1 \mathrm{mEquiv} \mathrm{L}^{-1} \mathrm{CaCl}_{2}$ feed solution was considered (mEquiv stands for milli-equivalents of charge; for a binary electrolyte the equivalent concentration is defined as $v_{i} z_{i}[i]$, with $v_{i}$ the number of ions $i$ released by the dissociation of a single electrolyte molecule, $z_{i}$ the charge number of ion $i$, and $[i]$ the molar concentration of ion $i$ ). The conical nanopores labelled "conical-divergent" (the pore tip is in contact with the high-pressure compartment filled with the feed solution) and "conical-convergent" (the pore base is in contact with the high-pressure compartment) exhibit filtration rectification properties since calcium chloride is found to be much better rejected as the pore tip is in contact with the high-pressure compartment. Similar qualitative conclusions about pressureinduced rectification properties of asymmetric conical nanopores were drawn (results not shown) from additional simulations performed with (i) wider conical nanopores (pores $\mathrm{F}$ and

G; see Table 1), (ii) different surface charge densities and (iii) feed solutions containing different types of electrolytes $\left(\mathrm{KCl}\right.$ and $\left.\mathrm{Na}_{2} \mathrm{SO}_{4}\right)$. On the other hand no rectification of the salt flux was observed with cylindrical and hourglass pores with constant surface charge density since none of them presents an overall asymmetry from the electrostatic point of view.

A rectification degree (RD) can be defined so as to quantify the filtration rectification properties of asymmetric conical nanopores. Let us define RD as the ratio between the salt concentrations at the outlet of convergent and divergent pores:

$$
\mathrm{RD}=\frac{c_{\text {outlet, convergent }}}{c_{\text {outlet, divergent }}}
$$

The rectification degree of the conical nanopores $\mathrm{B}$ and $\mathrm{C}$ (see Table 1) is shown in Figure 3. It is found to be dependent upon the driving force, similarly to what was already reported in the case of electrically-driven transport $[2,16]$. Strikingly, a significant rectification degree is observed ( $\mathrm{RD}>4$ for $\Delta P=50$ bar) although pores $\mathrm{B}$ and $\mathrm{C}$ geometry deviates only very 


\section{ACCEPTED MANUSCRIPT}

slightly from cylindrical geometry since the half cone angle $\alpha$ (see Figure 1), defined as $\alpha=\arctan \left(\frac{d_{\text {base }}-d_{t i p}}{2 L}\right)$, is only $0.23^{\circ}$.

The filtration rectification phenomenon observed with conical nanopores can be easily understood by the stronger co-ions (i.e. chloride ions) exclusion occurring as the pore tip is in contact with the feed solution (see the axial profiles of the co-ion concentration in Figure 4), which results from the higher pore volume charge density (in absolute value) in the pore-inlet region. The higher salt rejection obtained with the cylindrical pore compared with the conicalconvergent one (see Figure 2) can be justified by the same argument since the inlet diameter of the cylindrical pore is smaller than that of the conical-convergent one $(1.2 \mathrm{~nm}$ against 1.6 $\mathrm{nm}$; see Table 1). It is worth mentioning that in the case of single binary electrolytes it is usual to focus only on co-ions because they limit the overall salt transfer since the electrolyte solution exiting the nanopore must be electroneutral $\left(\sum_{i} c_{i, \text { outlet }} z_{i}=0\right)[45]$.

However, results reported in Figure 2 show that the hourglass-shape nanopore exhibits the best rejection performance among the different pores although its inlet diameter is wider than that of both the cylindrical and conical-divergent pores. This suggests that an additional phenomenon comes into play. Moreover, the dependence of RD towards the applied driving force $(\Delta P)$ highlighted in Figure 3 sheds light on the non-equilibrium nature of this phenomenon.

Under nanofiltration conditions, an electric field arises spontaneously through the nanopore as a response to the applied hydrostatic pressure gradient, so that no net electrical current flows through the nanopore at steady-state $\left(\sum_{i} \mathbf{j}_{i} z_{i}=0\right)$. In the case of nanopores without any electrostatic asymmetry (e.g. a cylindrical pore with a constant surface density) this electric field always drives the counter-ions towards the high-pressure side so as to ensure the electroneutrality of the pore-filling solution [45]. Szymczyk et al. showed that the (pressure- 


\section{ACCEPTED MANUSCRIPT}

induced) electric field arising through cylindrical nanopores having heterogeneous charge distributions can be strengthened, weakened or even reversed depending on the sign of the space charge density gradient through the nanopore [17]. Figure 5 shows an example of the axial profile of the (pressure-induced) electric field along the pore axis of the different nanopores (note that, as expected for the small pore sizes under consideration with respect to the Debye screening length, the radial variations of the electric field were found to be negligible). For the sake of clarity, the regions very close to the pore mouths, where the local electric field can reach very high values, are not shown in Figure 5 (see the supporting information for results over the entire pore length). Strikingly, the electric field has a different sign in the conical-divergent and conical-convergent nanopores. It results from the different sign of the space charge density gradient in the two systems. Indeed, the absolute value of the nanopore volume charge density $\left(\mathrm{C} \mathrm{m}^{-3}\right)$ decreases from the pore inlet to the pore outlet in the conical-divergent nanopore (i.e. from the pore tip to its base) whereas the opposite is true for the conical-convergent nanopore. This phenomenon also contributes to the filtration rectification properties of conical nanopores shown in Figure 2 and 3. As expected, the electric field in the hourglass pore changes sign in the middle of the pore $(z=50 \mathrm{~nm})$ because the sign of the charge gradient changes too. Interestingly, the electric field is found to be much stronger in the hourglass nanopore than in both the conical-divergent and conicalconvergent nanopores, which explains the better rejection performance of the hourglass nanopore reported in Figure 2. It results from the stronger charge gradient in the hourglass pore since the base/tip distance in this pore is twice as less as in the conical-divergent and conical-convergent nanopores (the pore length was set to $100 \mathrm{~nm}$ for all systems as indicated in Table 1). Otherwise stated, the magnitude of the electric field depends upon the cone angle $\alpha$ as already noticed by Tseng et al. in the case of electrically-driven ion transport [27]. It should be stressed, however, that if $\alpha$ is increased while keeping the tip diameter constant, then the co-ion exclusion in the pore inlet region is expected to be weakened since the base 


\section{ACCEPTED MANUSCRIPT}

diameter is larger. Our results therefore suggest that there is an optimal cone angle for which hourglass nanopores would perform best (this issue is out of the scope of the present study and will be addressed in a subsequent work).

In the last part of this work we wanted to check whether the improved nanofiltration performance of the hourglass-shape nanopore reported in Figure 2 was still observed under different conditions of surface charge density, pore size and electrolyte type. Since nanofiltration has been used in many numerous applications (e.g. desalination, solute concentration, water hardness lowering, etc.) for which substantially different rejection rates are sought for, we computed the pressure difference to be applied through nanopores with different properties in order to reach some target rejection rates. Results are shown in Figure 6. Interestingly, the driving force required to reach the target salt rejection is always found to be weaker as the separation is performed with hourglass nanopores, thus confirming the improved nanofiltration performance of these latter. These results are of great interest since they mean that nanofiltration could be operated with substantial energy savings by designing advanced nanoporous membranes with hourglass-shaped pores.

\section{Conclusion}

The nanofiltration performance of conical and hourglass-shape (double conical) nanopores was investigated by means of an approximate approach based on Poisson-Nernst-Planck theory and continuum hydrodynamics. Similarly to what was already reported for electricallydriven ion transport, conical nanopores were found to exhibit pressure-driven salt flux rectification properties, i.e. the salt flux is different if the solution is forced to flow through the nanopore from the base to the tip of from the tip to the base. These filtration rectification properties were explained by the different co-ion exclusion at the pore mouth and by the different (pressure-induced) electric field arising through the nanopores (notably it was shown that this latter changes sign as the electrolyte solution enters the nanopore by its base or its 


\section{ACCEPTED MANUSCRIPT}

tip). Hourglass-shape nanopores were found to outperform the separation performance of both cylindrical and conical nanopores, which was explained by the stronger electric field arising through hourglass-shape nanopores due to larger cone angles. The simulations performed in this work also suggest that membranes with hourglass-shaped nanopores could make the nanofiltration process more energy efficient by decreasing the driving force required to operate the separation.

\section{Appendix A. Supporting information}

Supplementary data associated with this article can be found in the online version.

\section{References}

[1] J. Cervera, B. Schiedt, R. Neumann, S. Mafé, P. Ramírez, Ionic conduction, rectification, and selectivity in single conical nanopores, J. Chem. Phys. 124 (2006) 104706.

[2] D. Constantin, Z. S. Siwy, Poisson-Nernst-Planck model of ion current rectification through a nanofluidic diode, Phys. Rev. E 76 (2007) 041202.

[3] S. W. Nam, M. J. Rooks, K. B. Kim, M. Rossnagel, Ionic field effect transistors with sub-10 nm multiple nanopores, Nano Lett. 9 (2009) 2044-2048.

[4] M. Ali, B. Yameen, J. Cervera, P. Ramírez, R. Neumann, W. Ensinger, W. Knoll, O. Azzaroni, Layer-by-layer assembly of polyelectrolytes into ionic current rectifying solid-state nanopores: insights from theory and experiment, J. Am. Chem. Soc. 132 (2010) 8338-8348.

[5] L. J. Steinbock, A. Lucas, O. Otto, U. F. Keiser, Voltage-driven transport of ions and DNA through nanocapillaries, Electrophoresis 33 (2012) 3480-3486.

[6] Y. L. Ying, J. J. Zhang, R. Gao, Y. T. Long, Nanopore-based sequencing and detection of nucleic acids, Angew. Chem., Int. Ed. 52 (2013) 13154-13161. 


\section{ACCEPTED MANUSCRIPT}

[7] N. Sa, W. J. Lan, W. Shi, L. A. Baker, Rectification of ion current in nanopipettes by external substrates, ACS Nano 7 (2013) 11272-11282.

[8] H. C. Zhang, Y. Tian, L. Jiang, Fundamental studies and practical applications of bioinspired smart solid-state nanopores and nanochannels, Nano Today 11 (2016) 61-81.

[9] D. Stein, M. Kruithof, C. Dekker, Surface-charge-governed ion transport in nanofluidic channels, Phys. Rev. Lett. 93 (2004) 035901.

[10] H. Daiguji, P. Yang, A. J. Szeri, A. Majumdar, Electrochemomechanical energy conversion in nanofluidic channels, Nano Lett. 4 (2004) 2315-2321.

[11] S. Bouranene, P. Fievet, A. Szymczyk, Investigating nanofiltration of multi-ionic solutions using the Steric, Electric and Dielectric Exclusion model, Chem. Eng. Sci. 64 (2009) 3789-3798.

[12] A. Fuliński, I. D. Kosińska, Z. Siwy, On the validity of continuous modelling of ion transport through nanochannels, Europhys. Lett. 67 (2004) 683-689.

[13] R. Karnik, C. Duan, K. Castelino, H. Daiguji, A. Majumdar, Rectification of ionic current in a nanofluidic diode, Nano Lett. 7 (2007) 547-551.

[14] P. Ramírez, V. Gómez, J. Cervera, B. Schiedt, S. Mafé, Ion transport and selectivity in nanopores with spatially inhomogeneous fixed charge distributions, J. Chem. Phys. 126 (2007) 194703.

[15] L. J. Cheng, L. J. Guo, Rectified ion transport through concentration gradient in homogeneous silica nanochannels, Nano Lett. 7 (2007) 3165-3171.

[16] N. R. Scruggs, J. W. F. Robertson, J. J. Kasianowicz, K. B. Migler, Rectification of the ionic current through carbon nanotubes by electrostatic assembly of polyelectrolytes, Nano Lett. 9 (2009) 3853-3859.

[17] A. Szymczyk, H. Zhu, B. Balannec, Pressure-driven ionic transport through nanochannels with inhomogeneous charge distributions, Langmuir 26 (2010) 12141220. 


\section{ACCEPTED MANUSCRIPT}

[18] A. Szymczyk, H. Zhu, B. Balannec, Ion rejection properties of nanopores with bipolar fixed charge distributions, J. Phys. Chem. B 114 (2010) 10143-10150.

[19] H. Zhu, A. Szymczyk, B. Balannec, On the salt rejection properties of nanofiltration polyamide membranes formed by interfacial polymerization, J. Membr. Sci. 379 (2011) 215-223.

[20] Z. Ható, M. Valiskó, T. Kristóf, D. Gillespie, D. Boda, Multiscale modeling of a rectifying bipolar nanopore: explicit-water versus implicit-water simulations, Phys. Chem. Chem. Phys. 19 (2017) 17816-17826.

[21] Z. S. Siwy, Ion-current rectification in nanopores and nanotubes with broken symmetry, Adv. Funct. Mater. 16 (2006) 735-746.

[22] J. Cervera, B. Schiedt, P. Ramírez, A Poisson/Nernst-Planck model for ionic transport through synthetic conical nanopores, Europhys. Lett. 71 (2005) 35-41.

[23] Y. He, D. Gillespie, D. Boda, I. Vlassiouk, R. S. Eisenberg, Z. S. Siwy, Tuning transport properties of nanofluidic devices with local charge inversion, J. Am. Chem. Soc. 131 (2009) 5194-5202.

[24] I. Vlassiouk, Z. S. Siwy, Nanofluidic diode, Nano Lett. 7 (2007) 552-556.

[25] Z. Siwy, I. D. Kosińska, A. Fuliński, C. R. Martin, Asymmetric diffusion through synthetic nanopores, Phys. Rev. Lett. 94 (2005) 048102.

[26] P. Jin, H. Mukaibo, L. P. Horne, G. W. Bishop, C. R. Martin, Electroosmotic flow rectification in pyramidal-pore mica membranes, J. Am. Chem. Soc. 132 (2010) 21182119.

[27] S. Tseng, S. C. Lin, C. Y. Lin, J. P. Hsu, Influences of cone angle and surface charge density on the ion current rectification behavior of a conical nanopore, J. Phys. Chem. C 120 (2016) 25620-25627.

[28] E. Weatherall, P. Hauer, R. Vogel, G. R. Willmott, Pulse size distributions in tunable resistive pulse sensing, Anal. Chem. 88 (2016) 8648-8656. 


\section{ACCEPTED MANUSCRIPT}

[29] Y. Zhang, G. C. Schatz, Conical nanopores for efficient ion pumping and desalination, J. Phys. Chem. Lett. 8 (2017) 2842-2848.

[30] D. Wang, M. V. Mirkin, Electron-transfer gated ion transport in carbon nanopipets, J. Am. Chem. Soc. 139 (2017) 11654-11657.

[31] W.R. Bowen, A.W. Mohammad, A theoretical basis for specifying nanofiltration membranes - dye/salt/water streams, Desalination 117 (1998) 257-264.

[32] X. Gong, J. Li, H. Lu, R. Wan, J. Li, J. Hu, H. Fang, A charge-driven molecular water pump, Nature Nanotechnol. 2 (2007) 709-712.

[33] R. Renou, A. Ghoufi, A. Szymczyk, H. Zhu, J.C. Neyt, P. Malfreyt, Nanoconfined Electrolyte Solutions in porous Hydrophilic Silica Membranes, J. Phys. Chem. C 117 (2013) 11017-11027.

[34] G. Hummer, J. C. Rasaiah, J. P. Noworyta, Water conduction through the hydrophobic channel of a carbon nanotube, Nature 414 (2001) 188-190.

[35] P. Ramírez, S. Mafé, V. M. Aguilella, A. Alcaraz, Synthetic nanopores with fixed charges: an electrodiffusion model for ionic transport, Phys. Rev. E 68 (2003) 011910.

[36] A. Szymczyk, M. Sbaï, P. Fievet, A. Vidonne, Transport properties and electrokinetic characterization of an amphoteric nanofilter, Langmuir 22 (2006) 3910-3919.

[37] L. Bocquet, E. Charlaix, Nanofluidics, from bulk to interfaces, Chem. Soc. Rev. 39 (2010) 1073-1095.

[38] S. Gravelle, L. Joly, F. Detcheverry, C. Ybert, C. Cottin-Bizonne, L. Bocquet, Optimizing water permeability through the hourglass shape of aquaporins, Proc. Natl. Acad. Sci. USA 110 (2013) 16367-16372.

[39] Y. Jiang, Y. Feng, J. Su, J. Nie, L. Cao, L. Mao, L. Jiang, W. Guo, On the origin of ionic rectification in DNA-stuffed nanopores: the breaking and retrieving symmetry, J. Am. Chem. Soc. 139 (2017) 18739-18746. 


\section{ACCEPTED MANUSCRIPT}

[40] L. Cao, W. Guo, W. Ma, L. Wang, F. Xia, S. Wang, Y. Wang, L. Jiang, D. Zhu, Towards understanding the nanofluidic reverse electrodialysis system: well matched charge selectivity and ionic composition, Energy Environ. Sci. 4 (2011) 2259-2266.

[41] Z. Zhang, X.Y. Kong, K. Xiao, Q. Liu, G. Xie, P. Li, J. Ma, Y. Tian, L. Wen, L. Jiang, Engineered asymmetric heterogeneous membrane: a concentration-gradient-driven energy harvesting device, J. Am. Chem. Soc. 137 (2015) 14765-14772.

[42] S. Basu, M.M. Sharma, An improved space-charge model for flow through charged microporous membranes, J. Membr. Sci. 124 (1997) 77-91.

[43] A. Szymczyk, P. Fievet, B. Aoubiza, C. Simon, J. Pagetti, An application of the space charge model to the electrolyte conductivity inside a charged microporous membrane, J. Membr. Sci. 161 (1999) 275-285.

[44] A. Szymczyk, C. Labbez, P. Fievet, B. Aoubiza, C. Simon, Streaming potential through multilayer membranes, AIChE J. 47 (2001) 2349-2358.

[45] A. Szymczyk, C. Labbez, P. Fievet, A. Vidonne, A. Foissy, J. Pagetti, Contribution of convection, diffusion and migration to electrolyte transport through nanofiltration membranes, Adv. Colloid Interface Sci. 103 (2003) 77-94. 
Table 1. Geometrical features of the various pores considered in this work. Inlet and outlet refer to the pore mouth in contact with the high pressure and low pressure compartments, respectively. The pore length $(L)$ and mouth diameters $\left(d_{\text {inlet }}\right.$ and $\left.d_{\text {outlet }}\right)$ were chosen in a range that is relevant with the structural features of actual nanofiltration membranes $[19,31]$.

\begin{tabular}{|c|c|c|c|c|c|c|}
\hline Pore geometry & $\begin{array}{c}L \\
(\mathrm{~nm})\end{array}$ & $\begin{array}{l}d_{\text {inlet }} \\
(\mathrm{nm})\end{array}$ & $\begin{array}{c}\boldsymbol{d}_{(\mathrm{z}=50 \mathrm{~nm})} \\
(\mathrm{nm})\end{array}$ & $\begin{array}{l}d_{\text {outlet }} \\
(\mathbf{n m})\end{array}$ & $\begin{array}{l}\text { Inner pore surface area } \\
\qquad\left(\mathbf{n m}^{2}\right)\end{array}$ & $\begin{array}{c}\text { Pore volume } \\
\left(\mathbf{n m}^{3}\right)\end{array}$ \\
\hline A: cylindrical & 100 & 1.2 & 1.2 & 1.2 & \multirow{4}{*}{377} & 113 \\
\hline B: conical-divergent & 100 & 0.8 & 1.2 & 1.6 & & \multirow{3}{*}{117} \\
\hline C: conical-convergent & 100 & 1.6 & 1.2 & 0.8 & & \\
\hline D: hourglass & 100 & 1.6 & 0.8 & 1.6 & & \\
\hline E: cylindrical & 100 & 2.4 & 2.4 & 2.4 & \multirow{4}{*}{754} & 452 \\
\hline F: conical-divergent & 100 & 1.6 & 2.4 & 3.2 & & \multirow{3}{*}{469} \\
\hline G: conical-convergent & 100 & 3.2 & 2.4 & 1.6 & & \\
\hline H: hourglass & 100 & 3.2 & 1.6 & 3.2 & & \\
\hline
\end{tabular}


$L=100 \mathrm{~nm}$

Cylindrical pore

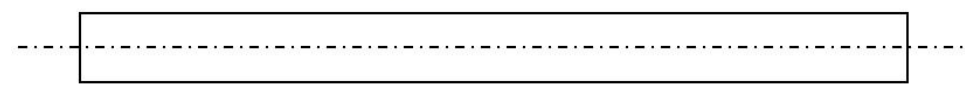

Conical-divergent pore

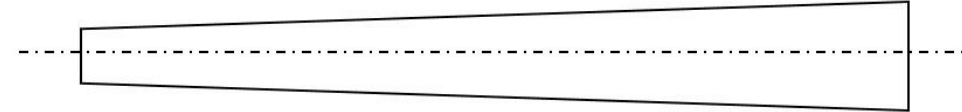

Conical-convergent pore

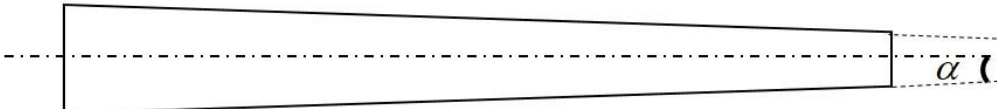

Hourglass pore
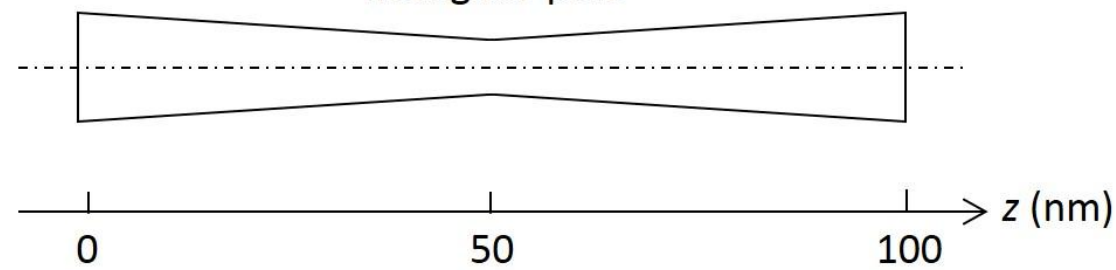

Inlet Outlet

Figure 1. Schematic of the different pore shapes (not to scale) considered in this work. 


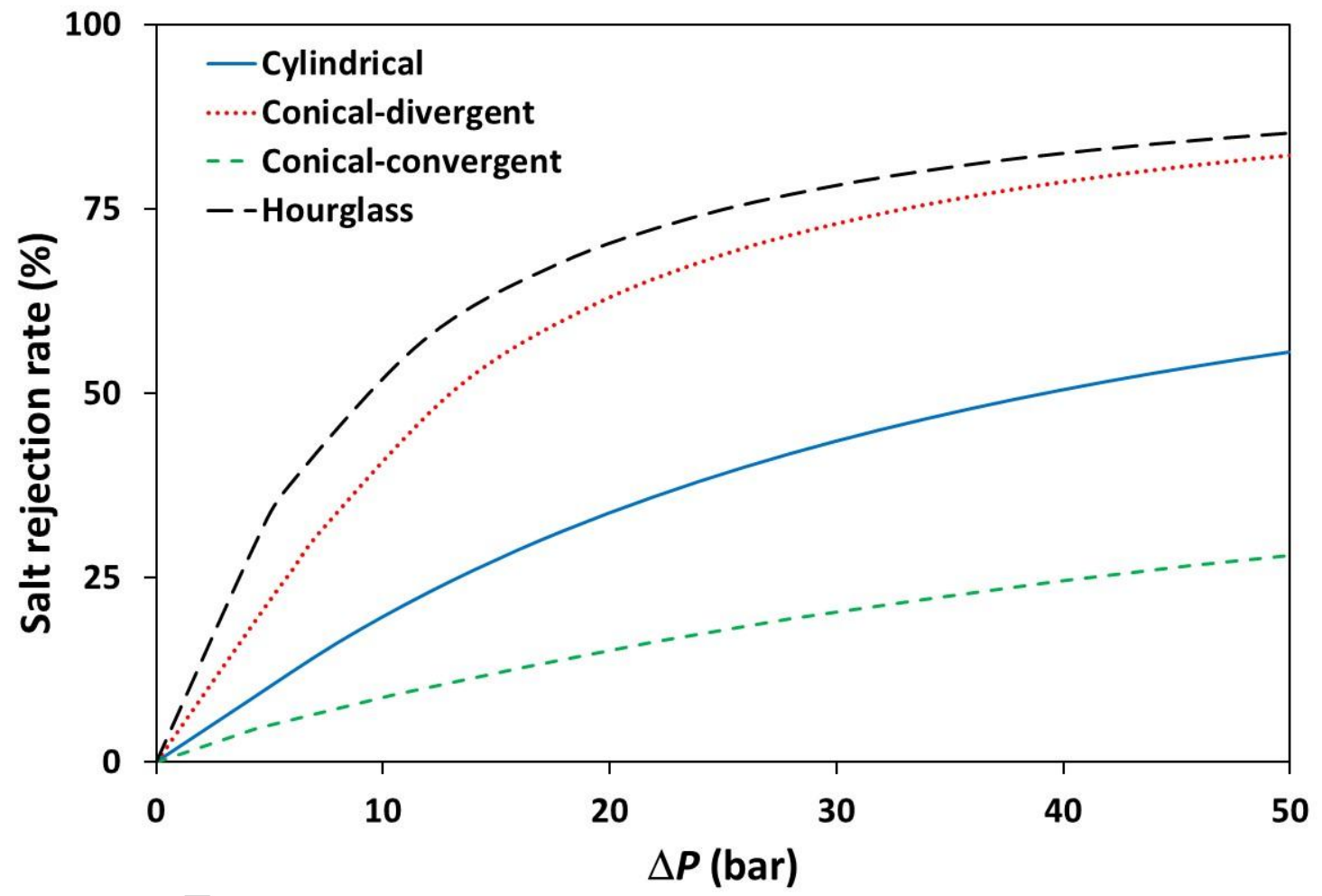

Figure 2. Salt rejection rate vs. pressure difference $(\Delta P)$ through nanopores with different geometries. Simulations were carried out with pores A-D (see Table 1), a surface charge density $(\sigma)$ of $-1 \mathrm{mC} \mathrm{m}^{-2}$ and a $1 \mathrm{mEquiv}^{-1} \mathrm{CaCl}_{2}$ feed solution. 


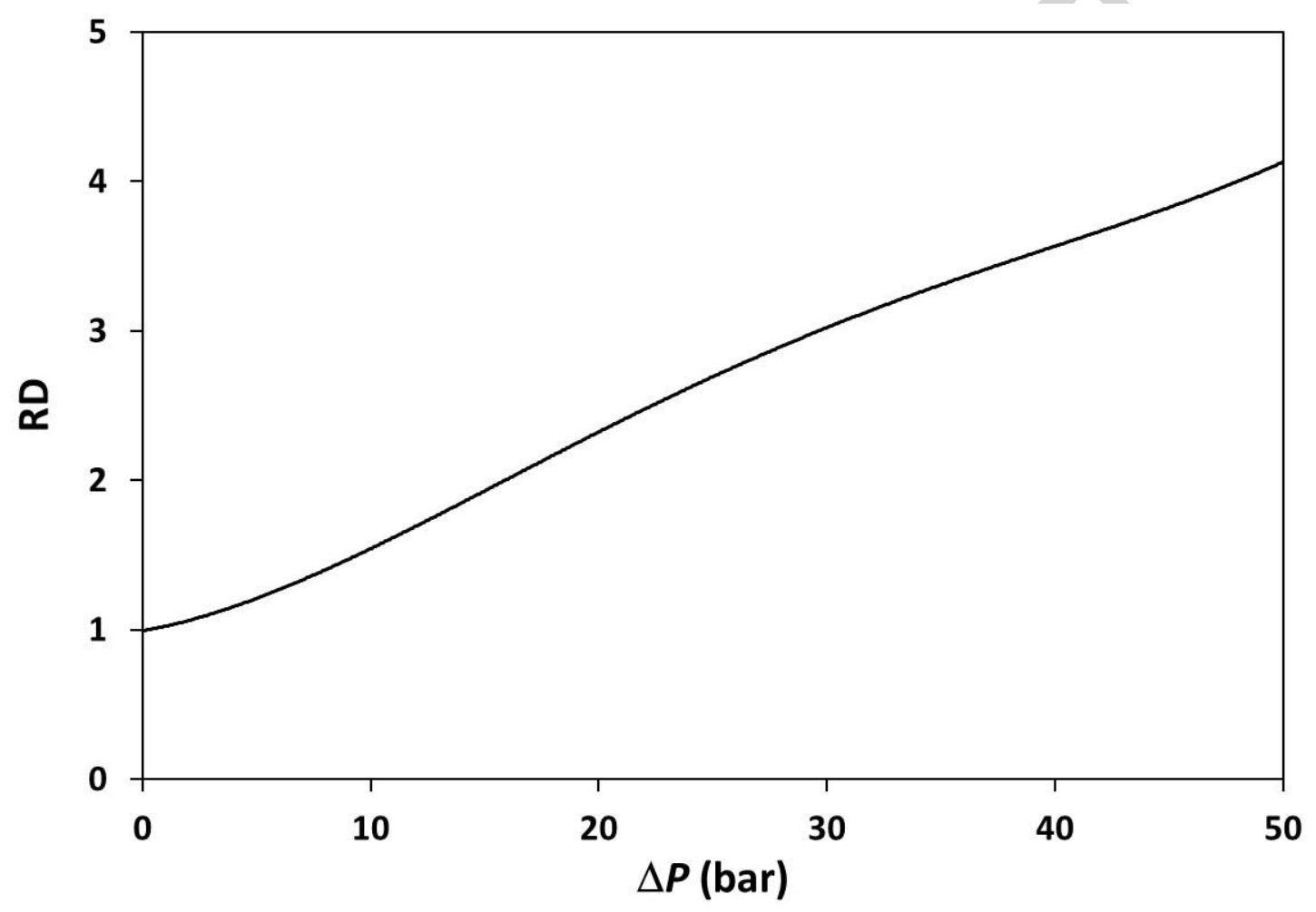

Figure 3. Rectification degree $(\mathrm{RD})$ vs. pressure difference $(\Delta P)$ for the conical nanopores $\mathrm{B}$ and $\mathrm{C}$ (see Table 1). Simulations were carried out with $\sigma=-1 \mathrm{mC} \mathrm{m}^{-2}$ and a $1 \mathrm{mEquiv} \mathrm{\textrm {L } ^ { - 1 }}$ $\mathrm{CaCl}_{2}$ feed solution. 


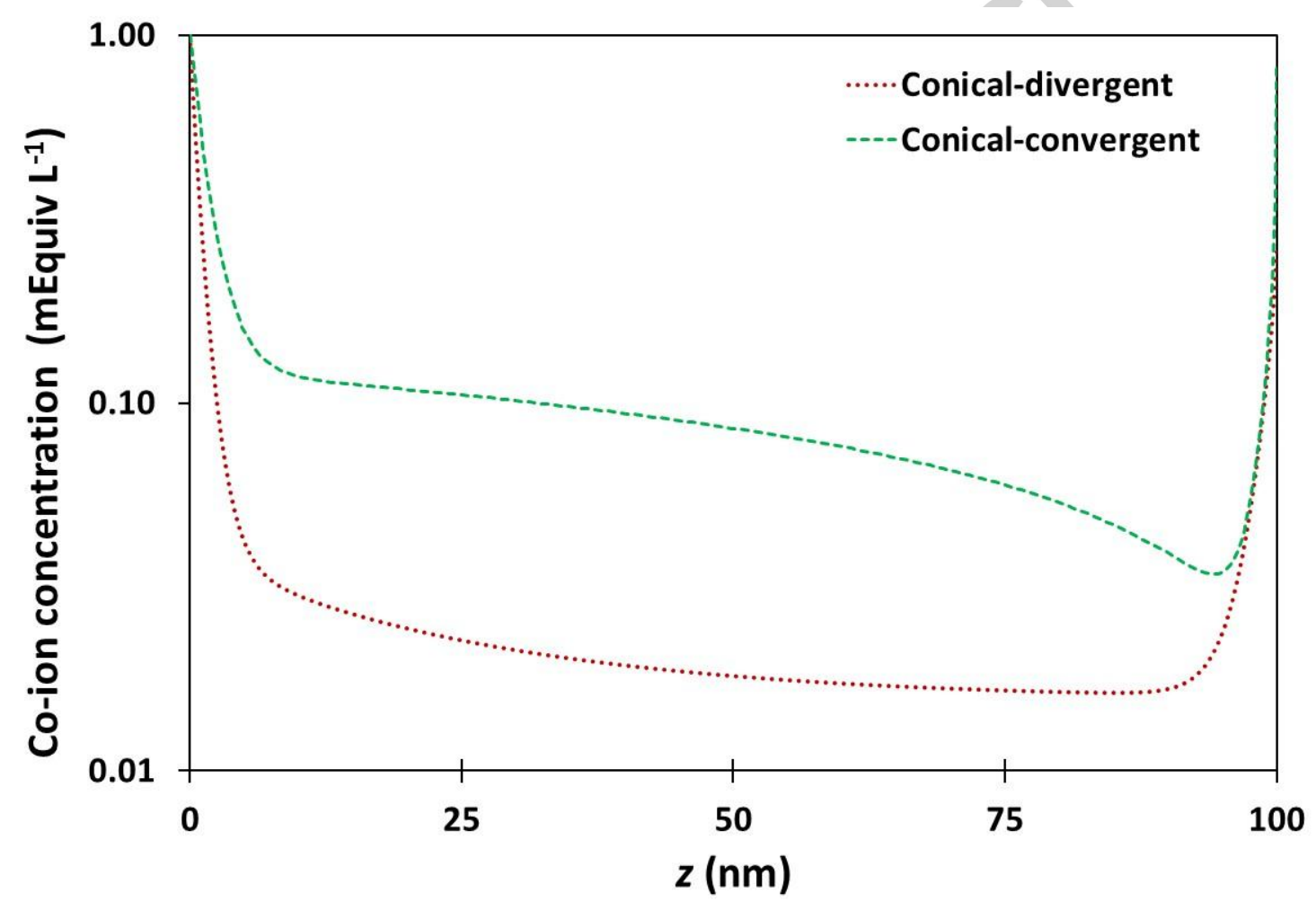

Figure 4. Axial profile of the co-ion concentration inside the conical nanopores $B$ and $C$ (see Table 1). Simulations were carried out with $\sigma=-1 \mathrm{mC} \mathrm{m}^{-2}$ and a $1 \mathrm{mEquiv} \mathrm{L}^{-1} \mathrm{CaCl}_{2}$ feed solution. 


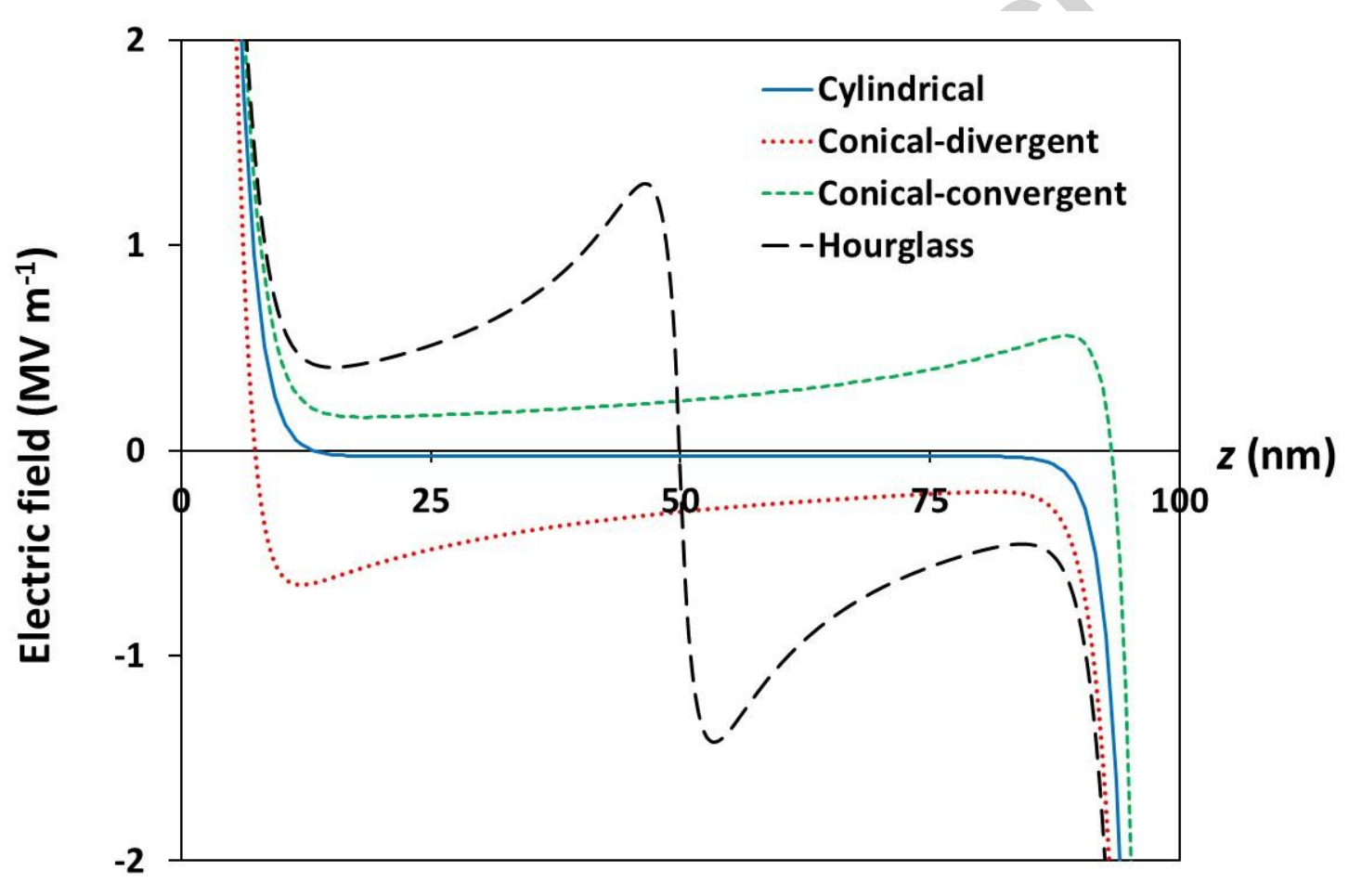

Figure 5. Axial profile of the electric field inside nanopores with different geometries. Simulations were carried out with pores A-D (see Table 1), $\sigma=-1 \mathrm{mC} \mathrm{m}^{-2}$ and a $1 \mathrm{mEquiv} \mathrm{L^{-1 }}$ $\mathrm{CaCl}_{2}$ feed solution. $\Delta P$ was about 30 bar through the different nanopores. 

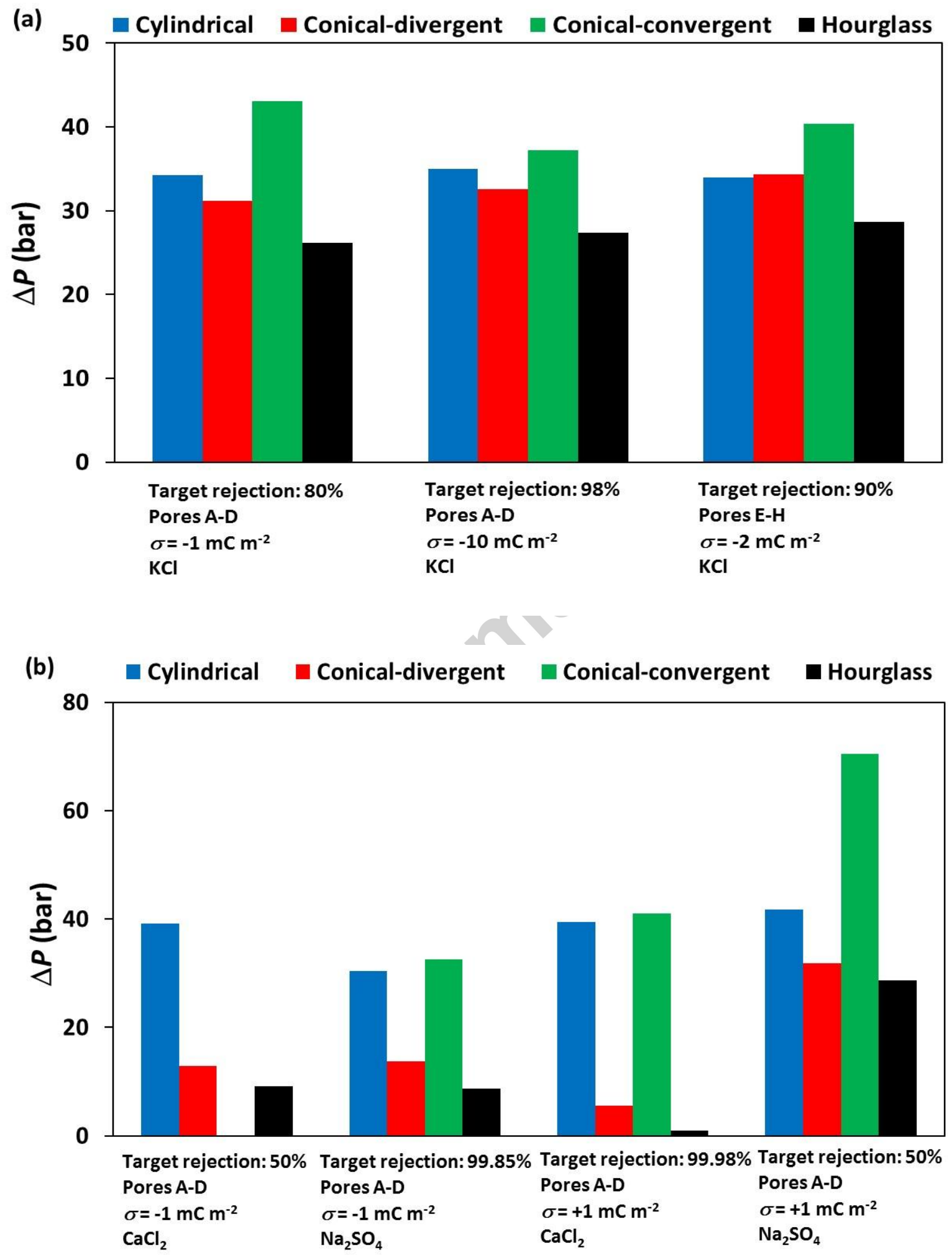


\title{
ACCEPTED MANUSCRIPT
}

Figure 6. Pressure difference $(\Delta P)$ required to reach target rejection rates. (a) Performance of pores A-H (see Table 1) with different surface charge densities $(\sigma)$ towards $\mathrm{KCl}$ solutions; (b) Performance of pores A-D with different $\sigma$ towards asymmetric electrolytes (note that the target $\mathrm{CaCl}_{2}$ rejection cannot be reached with the negatively charged conical-convergent nanopore). All simulations were performed with $1 \mathrm{mEquiv} \mathrm{L}^{-1}$ feed solutions.

\section{Supporting Information}

\section{Nanofiltration performance of conical and hourglass nanopores}

\author{
Béatrice Balannec ${ }^{1}$, Aziz Ghoufi ${ }^{2}$, Anthony Szymczyk ${ }^{1}$ * \\ ${ }^{1}$ Univ Rennes, Ecole Nationale Supérieure de Chimie de Rennes, CNRS, ISCR (Institut des \\ Sciences Chimiques de Rennes) - UMR 6226, F-35000 Rennes, France \\ ${ }^{2}$ Univ Rennes, CNRS, IPR (Institut de Physique de Rennes) - UMR 6251, F-35000 Rennes, \\ France
}

*E-mail: anthony.szymczyk@univ-rennes1.fr 


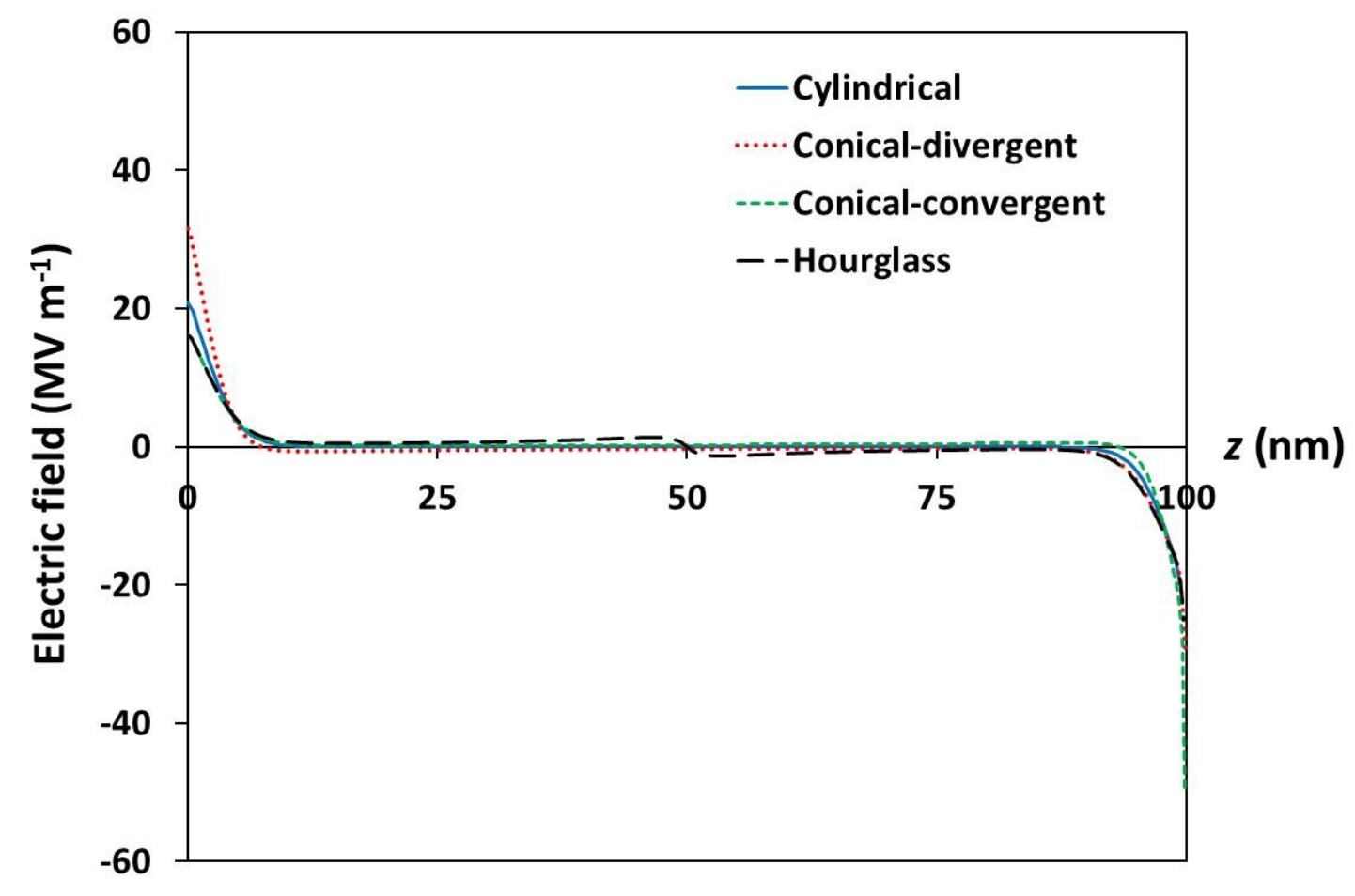

Figure S1. Axial profile of the electric field inside nanopores with different geometries. Simulations were carried out with pores A-D (see Table 1), $\sigma=-1 \mathrm{mC} \mathrm{m}^{-2}$ and a $1 \mathrm{mEquiv} \mathrm{\textrm {L } ^ { - 1 }}$ $\mathrm{CaCl}_{2}$ feed solution. $\Delta P$ was about 30 bar through the different nanopores. 


\section{ACCEPTED MANUSCRIPT}

\section{Highlights:}

- Pressure-driven transport through conical and hourglass-shape pores is investigated

- Conical nanopores exhibit nanofiltration rectification properties

- Rectification result from different co-ion exclusion and induced electric field

- Hourglass-shape nanopores exhibit improved separation performance

- Hourglass-shape nanopores could make nanofiltration more energy efficient 